\title{
Tracking patients with advanced kidney disease in last 12 months of life
}

\section{ABSTRACT}

Background: There is increasing recognition of the clinical need for timely and coordinated supportive and palliative care for those with terminal organ failure.

Objective: To describe symptoms, quality of life and supportive care needs in the anticipated 12-month period prior to death in adults with chronic kidney disease stages 4 or 5 .

Method: An observational, prospective, longitudinal design was used to follow 19 patients. The measures used were the Chronic Kidney Disease-Symptom Burden Index (CKD-SBI), the Australian Karnofsky Performance Scale (AKPS), the Functional Assessment of Chronic illness Therapy Palliative-14 (FACIT PAL-14), the Assessment of Quality of Life 6 Dimensions (AQoL-6D), and the Sheffield Profile for Assessment and Referral for Care (SPARC). Data were collected at study entry and 3 monthly until death or study end. Results: Patients' median age was 78 years (range 42-90), most were male (63\%), 10 were receiving dialysis, and 7 died during the study. The most prevalent symptoms reported differed from those most troublesome. The median AKPS score did not change over time (60). Quality of life remained steady over time (FACIT-PAL median range: 43.5-46; AQoL6D means range: 0.66 [SD 0.19] to 0.75 [SD 0.2]). Supportive care needs were few.

Conclusion: We found a substantial symptom burden and slow functional decline in these patients. Regular assessment of both symptoms and QOL is warranted particularly if clinical experience indicates that the person is likely to be in their last year of life. Integrated supportive care programs could assist with easing symptom burden during this time.

Key Words: Chronic kidney disease; End-of-life; Supportive care; Symptom experience; Mapping outcomes 


\section{INTRODUCTION}

Palliative care is increasingly provided earlier in the cancer trajectory to substantially reduce symptom burden and to have a seamless pathway to end of life care (Hui \& Bruera 2016), but the concept has not been translated to those with terminal organ failure (Bostwick et al. 2017). In chronic kidney disease (CKD), Davison et al. (2015) argues the case not only for improvements in the quality of care but also for the integration of palliative care principals into usual CKD care through reforms of health systems and policies. There is also a lack of clarity about terms (conservative, supportive and palliative care - see Murtagh et al. 2016). The nature of palliative care in advanced stages of CKD is confounded by the heterogeneity of the patient population, a substantial symptom burden (Almutary et al. 2016), the availability of dialysis as a care pathway, and the option to cease dialysis (Murtagh et al. 2016). In Australia, for instance, withdrawal from haemodialysis is the leading cause of death in people with end stage kidney disease receiving renal replacement therapy (ANZDATA registry 2017). Paradoxically palliative care is often unable to respond fast enough with average time to death very short (7-10 days) unless withdrawal from dialysis is pre-planned. Conversely of those who opt for conservative care (often older and greater comorbid diseases), $20 \%$ are likely to still be alive three years later with relatively few patients switching to dialysis during that time (Morton et al. 2016). The CKD population has, therefore, different trajectories towards end of life and all need (at some point) kidney supportive care.

The World Health Organization (WHO) defines palliative care as an approach that improves the quality of life (QOL) of persons with life limiting illnesses, and their families, 'through the prevention and relief of suffering through early identification and impeccable assessment and treatment of pain and other problems, physical, psychosocial and spiritual' (World Health 
Organization 2016). However, the term palliative care also denotes specific health services (Singer et al, 2016). Palliative care is provided in acute hospital settings, the community (which includes residential aged care facilities and people's homes), and specialist palliative care units and hospices. Globally the provision of effective and sustainable palliative care services is of increasing concern for governments with the WHO urging countries to develop, strengthen and implement palliative care policies across all levels of health care, and to integrate evidence-based, cost effective and equitable palliative care services (PCS) (World Health Organization Executive Board 2013).

In a large retrospective Australian study of people who had accessed PCS ( $\mathrm{n}=12,817)$, those with renal failure $(8.9 \%$ of the sample; CKD stage and modality type were not reported) received a median of six days of PCS as compared to 30 days received by those with a cancer diagnosis (56\% of the total sample) (Rosenwax et al. 2016). With the slow progression of CKD accompanied by acute and unpredictable exacerbations of clinical problems (Ngu et al. 2017), concomitant comorbid conditions, and multiplicity of treatment pathways (conservative, renal replacement therapies), flexible and extended palliative care support may be required. The current models of PCS which tend to focus on the later stages of decline may not be a good fit for the varied population with CKD.

To better understand patients' use and experiences of health services, prospective assessment of patterns of care in the last months of life is needed. Abstracted from a larger study which sought to track patients with advanced CKD and their health service utilisation over 12 months prior to their anticipated death, this paper reports on their symptoms, quality of life and supportive care needs over that period. A secondary aim was to report on the feasibility of following these patients for 12 months. 


\section{METHODS}

\section{Design}

The study design was prospective, longitudinal and observational. Data were collected at regular intervals from multiple sources for a 12 month period, or until patient death. Patients' palliative phase, symptom experience and functional status were collected at study entry (Time $0[\mathrm{~T} 0]$ ) and at monthly intervals for 12 months (T1 to $\mathrm{T} 12$ ) by phone or face-to-face. Patients' QOL, experiences of care and supportive care needs were collected face-to-face at T0 and then three monthly to study end (T3, T6, T9 and T12). Clinical and administrative health data were collected throughout the study period. Caregivers' demographic and comorbidity details were self-reported by the carers at baseline (T0) only. After study entry, with the patient's permission, a caregiver could respond for the patient if the patient was unable to do so due to illness. This study received ethics approval (HREC/14/QRBW/171). Patients and carers (i.e. dyads) volunteered to participate and all received study information. Informed consent was obtained prior to the commencement of the study.

\section{Setting and sample}

Patients, recruited from a large public hospital renal service in Brisbane, Australia from February 2015 to January 2016, were first identified by a nephrologist (HH, AK or SR). Included patients were English speaking adults ( $\geq 18$ years) with Stages 4-5 Chronic Kidney Disease (CKD) and a prognosis of $<12$ months (Clinician identified by answering 'no' to the surprise question, "would you be surprised if the patient died in the following 12 months?") and were cognitively sound and competent to give informed consent. The "surprise question" demonstrates good reliability with CKD end of life prognostication (Javier et al 2017). Patients were excluded if they had extreme psychological or social distress that would prevent them from completing interviewer administered surveys (determined by clinical 
and/or research staff), died within 48 hours of qualifying for the study, or resided $>2$ hours' drive from the recruitment site. This was because face-to-face interviews were required to visually determine functional performance via the Australian-modified Karnofsky Performance Scale (AKPS) and to maintain rapport with participants over 12 months.

Caregivers were English speaking adults ( $\geq 18$ years) who were cognitively sound and competent to give informed consent (identified by clinical and/or research staff). Caregivers were excluded if they had expressed distress to either the clinical or the research team. One member of the research team was responsible for all data collection to maintain consistency.

\section{Data sources and tools}

Data were collected from: (1) patient self-report outcome measures, (2) clinical assessment measures, and (3) hospital records. Measures were specifically designed for use with this population, and were valid and reliable. Physical functioning was interviewer-rated using the AKPS (Abernethy et al. 2005) which measures the patient's overall physical functioning status $(0=$ dead to $100=$ normal). The AKPS is used by all services involved in the Palliative Care Outcomes Collaboration (PCOC) (http://www.pcoc.org.au/), an Australian initiative to understand the variation in clinical outcomes for patients being cared for by specialist palliative care as an inpatient or at home with community services.

Patients' self-reported symptom experiences were captured using the Chronic Kidney Disease-Symptom Burden Index (CKD-SBI) (Almutary et al. 2015). This instrument assesses 32 symptoms across four dimensions (prevalence, distress, severity and frequency) although in our study we removed 1 symptom (nocturia). The scores in each dimension are summed to create an overall symptom burden index. 
Patients self-reported quality of life was captured using the Functional Assessment of Chronic Illness Therapy Palliative-14 item survey (FACIT Pal-14) (Zeng et al. 2013) and the Assessment of Quality of Life-6 Dimensions (AQoL 6D) (Richardson et al. 2012). The FACIT Pal-14 captures quality of life specific for palliative patients relating to physical, social/family, emotional and functional wellbeing; it is self-rated with responses from 0 (not at all) to 4 (very much). The AQoL 6D is a preference-based 20-item utility instrument designed because of the perceived limitations in other quality of life measures (Richardson et al. 2012). This instrument covers six quality of life dimensions involving independent living, relationships, mental health, coping pain and senses, and results can be compared to a normal healthy Australian population.

Patients' supportive care needs were assessed using the Sheffield Profile for Assessment and Referral for Care (SPARC) (Hughes et al. 2013), which is a 45-item, nine dimensional screening tool to assess the supportive/palliative care needs of patients with advanced illnesses regardless of diagnosis. Dimensions include communication and information, physical symptoms, psychological issues, religious and spiritual issues, independence and activity, family and social issues and treatment and personal issues. To avoid overlap with the AKPS and to reduce burden on patients, a modified version of SPARC (reducing the tool from 45 to 17 items) was used for this study. The dimensions included in this modified version of SPARC were communication and information issues and personal issues (dichotomous response of yes or no), as well as ratings from 0 (not at all) to 3 (very much) of distress or bother relating to psychological issues, religious and spiritual issues, family and social issues, and treatment issues. The interviewer also rated patients against the palliative 
care phase classification (Eagar et al. 2004) to provide a clinical indication of their stage of illness $($ stable $=1$, unstable $=2$, deteriorating $=3$, terminal $=4$ and bereaved $=5$ ).

Patients' sociodemographic information was self-reported and also collected from hospital records (CKD stage and comorbidities [using the Charlson Comorbidity Index; Charlson et al. 1994]). Caregivers self-reported their demographic and comorbidity data.

\section{Analysis}

Unique study codes were assigned to the participants on study entry. Descriptive statistics and frequency distributions were generated from the caregivers' demographic data and patients' demographic and clinical characteristics using statistical software; IBM SPSS 21. The patients' self-reported symptom severity and bothersome scores (captured using the CKD-SBI) were combined to produce the patients' most troublesome symptoms.

\section{RESULTS}

Treating nephrologists identified 49 potentially eligible patients; 32 were approached. Twenty-two patients provided written consent (69\%) and 13 nominated a caregiver to a participate; nine caregivers were approached and eight consented (89\%) (see Figure A1 [attached file] for more recruitment details). With minimal attrition (two patient/carer dyads and another patient withdrew), the final sample comprised $n=19$ patients and $n=6$ caregivers.

\section{Participant Profiles}

At study entry, the patients' median age was 78 years (range $42-90)$, most were male $(63 \%)$, one identified as being of Aboriginal origin (5\%), 10 (53\%) were receiving dialysis, and few (16\%) lived alone. The dialysis patient group were slightly younger (median age 74 years) 
and this group had all of the patients aged $<60$ years $(n=4)$ and fewer in the 80 years and older age range than the non-dialysis group. During the study period, six patients $(32 \%)$ died of their illness (one patient/carer dyad died in a car accident). Caregivers were slightly younger than patients (median 74 years; range 49-82), most were married (67\%) and most (50\%) were the spouse/partner of the patient. Two caregivers were patients' offspring. All participants had limited financial resources. The three most common comorbidities across patients and carers were cardiovascular disease $(84 \% ; 17 \%)$, hypertension $(79 \% ; 17 \%)$ and diabetes $(53 \% ; 17 \%)$ respectively. As expected, patients' median comorbidity index (Charlson et al. 1994) was higher than that of their carers (8 versus 5). Additional information of the patients' demographic profiles, disease stage and comorbidity profile can be found in Table 1.

\section{Symptoms}

While fatigue, dry skin, dry mouth, and bone or joint pain were the most prevalent symptoms across all time-points (see Table A1 in Attached file for full details), the most troublesome symptoms were sex and sleep problems, muscle soreness, constipation and breathlessness (see Table A2 for details). Furthermore, sleep problems, fatigue, numb and tingling feet, and muscle soreness were the most frequent symptoms reported (see Table A3 for details).

Tables A4 and A5 report the scores for severity and distress respectively. However, it must be noted that most patients declined to respond to both 'sex' related questions hence biasing the median scores of these two items across all time points.

Combined CKD-SBI changed little over time (median 13.48 at T0 to 14.95 at T12), although the scores ranged widely (1.47 to 39.76 at T0 and 2.25 to 27.45 at T12). Patients' not receiving dialysis had a higher CKD-SBI score over time which may reflect the age 
differences between the two groups. Figure 1 displays CKD-SBI scores at each time point by treatment group.

\section{Functional Status, Palliative Illness Phase and Quality of Life}

Most patients' physical functioning (AKPS) remained relatively high during the study (median 60: range 30-70). However, some patients delayed data collection (approximately $26 \%$ of data collection points) until such a time as they felt less unwell or were available for interview, thus potentially impacting on these and other measured outcomes. All 19 patients' palliative care phase was assessed as stable at baseline, and the median over time remained stable (range 1-3).

Collectively, patients expressed reasonably good and steady QOL throughout the study period with FACIT-Pal-14 median scores of 45 at each time point (range 20-56) (possible range 0-56, the higher the score the better the QOL). Similarly, AQoL-6D scores remained largely unchanged with means 0.66 (SD 0.19) to 0.75 (SD 0.2) over time. Notably, the independent living dimension score was much lower than the utility score and other dimensions (see Table 2).

Few supportive care needs as measured by SPARC were reported. Feeling unable to concentrate, lonely, and that everything is an effort were persistently bothersome to patients. At T6 (6 months after study entry) these three factors were reported by $30 \%$ of patients which was slightly higher than at baseline (T0) or at study end (T12) (21\% and 29\% respectively). This may be due to some patients being closer to death at T6 and thus were not alive nine and/or 12 months later (T9 and/or T12). 


\section{DISCUSSION}

This study was undertaken to examine symptom burden, physical functioning and QOL in the anticipated last year of life of those with advanced CKD either receiving dialysis or not.

Despite the small sample, this study highlights the heterogeneous patient population and their multiple care pathways experienced in clinical practice. Overall we found that while symptom burden was high, physical functioning and QOL remained relatively unchanged despite high comorbid loads. These findings document the slow and long decline in health experienced in those with advanced CKD regardless of whether they are receiving dialysis or not. These are also important points of differentiation between advanced CKD and terminal stages of organ failure or cancer where i) unlike cancer, CKD is never cured; ii) lifesustaining organ replacement therapy is available in end stage kidney disease, unlike other terminal organ failure; and iii) the differing patterns of functional decline and dependence is reflective of advanced CKD heterogeneity (Brady et al. 2017).

During the last decade, studies have reported that symptom burden experienced by patients with advanced CKD is considerable (Murtagh et al. 2007, Almutary et al. 2013) and similar to or greater than other chronic diseases including cancer (Solano et al. 2006, Bostwick et al. 2017). Prevalence of pain, fatigue and breathlessness was high in our study although sexual and sleep problems were more troublesome. Almutary et al. (2016) caution that highly prevalent symptoms may not be the most severe and that multidimensional assessment of a range of symptoms is required in those with CKD. We also found that the total symptom burden index remained relatively constant over the 12 month period which probably indicates both the unremitting persistence of multiple symptoms and the possibility of ineffective symptom management in our sample. We also found, unlike Almutary et al. (2016), but mirroring results of Tan et al. (2017), that the non-dialysis patients reported a higher 
symptom burden index score than those receiving dialysis. While the sample size of this study was too small to draw any conclusions, this may have been due to the higher comorbidities and older age in the non-dialysis group.

Of surprise, physical functioning appeared to remain relatively stable throughout the study period although AKPS scores were not captured close to death as these were not recorded in the clinical records reviewed. Moreover, some dips in functionality may not have been captured due to patients requesting data collection be rescheduled, and carers did not report reduced functional abilities. Even so, similar to our study, Tan et al. (2017) found that patients' functional status was relatively high at study entry and did not change greatly over time (6 months). However, the Tan et al. (2017) cohort, although having Stage 5 CKD, were not selected due to $\mathrm{a}<12$ month prognosis suggesting this may not be a unique outcome for patients in their last year of life and may reflect the greater frailty of patients in advanced stages of CKD.

People with CKD are known to have lower health related QOL (Webster et al. 2017); hence it was unsurprising that our findings concur, and, indeed were substantially lower than healthy population norms (Maxwell et al. 2016). Even so over 12 months the patients' QOL scores remained relatively stable, and this result may have been due to adaptation to living with a low QOL, that is, a response shift in a person's perception that their QOL has become their new normal (Schwartz \& Sprangers 1999). We found that the independent living sub-score was more than two standard deviations lower in this cohort than population norms (Maxwell et al. 2016). This, in part, may be due to the overall median age of the cohort coupled with the typical complications and mobility dysfunction experienced by patients with advanced CKD (e.g. pain). While the score for independent living was low, patients did manage to 
independently attend to their basic activities of daily living, such as dressing showering and other self-care activities but little else. Patients may have become accustomed to reduced functional abilities which could also explain why fewer supportive care needs were reported. Engagement in normal family and social activities outside of the home during the last year of life may have been affected due to the reduced ability to undertake these independently however the other QOL dimensions assessed do not capture these aspects. Providing effective symptom management may be one strategy to improve QOL and to reduce the social isolation.

\section{Strengths and limitations}

A major strength of this study is its prospective, longitudinal design. We report on patients' troublesome symptoms, physical functioning, palliative care phase, quality of life, and supportive care needs during the study period. As the study sample was small and recruited from a single kidney health service, the generalisability of the findings is limited. Even though the patients in this study were chosen due to their limited prognosis, relatively few patients died as results of illness during the study timeframe $(n=6$; another died in an accident). Comparison of this study's findings to previous studies (of CKD patients nearing the end of life) should also be treated with caution as previous studies may have defined their study population differently, such as persons in the last three or six months of life. Despite these limitations, the findings of this study provide insight into the patients' illness experience, QOL and supportive care needs. Lastly, we have also confirmed the feasibility of the study design, and the patient and carer acceptability of researching this group at a vulnerable time of life. The minimal attrition experienced during this study adds credibility to the study. 


\section{CONCLUSION}

We were able to track symptom experience, physical functioning and QOL in people identified with a high likelihood to be in their last year of life. In the face of high symptom burden, structured programs that integrate SPC much earlier into usual CKD clinical care may help to ease individuals' symptoms, sustain independent and social functionality and lead to improved QOL. Future larger, multisite studies can use these methods to track patients nearing end of life to provide further evidence of the complex renal and palliative care needs of these patients. In addition, qualitative studies may assist with exploring renal supportive care needs overtime as functional, QOL, and existential issues arise closer to death.

\section{Application to Practice}

There are several implications for practice arising from this study. First routine assessment of symptoms and functional performance using short simple tools such as the patient selfreported POS-S renal (Davison et al. 2015) for symptoms and clinician determined Karnofsky performance scale, ought to be conducted on all patients with advanced CKD (i.e. stages 4 and 5) whether on dialysis or not. Given the slow decline in trajectory, if the patient is stable, then a third monthly assessment would be beneficial for patients so that timely interventions (both pharmacological and non-pharmacological) can be commenced. Lastly, referral to or having specialist palliative care member in integrated into the renal team is likely to deliver benefits to patients through easing symptom burden in the last year of life. 


\section{References}

Abernethy A., Shelby-James T., Fazekas B., et al. (2005). The Australian-modified Karnofsky Performance Status (AKPS) scale: A revised scale for contemporary palliative care clinical practice. BMC Palliative Care 4(7): 1-12.

Almutary H., Bonner A., Douglas C. (2013). Symptom burden in chronic kidney disease: A review of recent literature. Journal of Renal Care 39(3), 140-150.

Almutary H., Bonner A., Douglas C. (2015). Arabic translation, adaptation and modification of the Dialysis Symptom Index for chronic kidney disease stages four and five. BMC Nephrology 16, 36.

Almutary H., Douglas C. Bonner A. (2016). Which patients with chronic kidney disease have the greatest symptom burden? A comparative study of advanced CKD stage and dialysis modality." Journal of Renal Care 42(2), 73-82.

ANZDATA Registry. (2017). 39th Report, Chapter 3: Mortality in End Stage Kidney Disease. Australia and New Zealand Dialysis and Transplant Registry, Adelaide, Australia.

Bostwick D., Wolf S., Samsa G., et al. (2017). Comparing the palliative care needs of those with cancer to those with common non-cancer serious illness." Journal of Pain and Symptom Management. 53(6), 1079-1084.

Brady B., Redahan L., Donohoe C.L., et al. (2017). Renal patients at end of life: A 5-year retrospective review. Progress in Palliative Care 25(5), 224-229.

Charlson M., Szatrowski T.P., Peterson J., et al. (1994). Validation of a combined comorbidity index." Journal of Clinical Epidemiology 47(11), 1245-51.

Davison S.N, Levin A., Moss, A.H., et al. (2015). Executive summary of the KDIGO controversies conference on supportive care in chronic kidney disease: Developing a road map to improve quality care. Kidney International 88(3), 447-459.

Eagar K., Gordon R., Green J., et al. (2004). An Australian casemix classification for palliative care: Lessons and policy implication of a national study. Palliative Medicine 18(3), 227-233.

Hughes P., Ahmed N., Winslow M., et al. (2013). Consumer views on a new holistic screening tool for supportive and palliative-care needs: Sheffield Profile for Assessment and Referral for Care (SPARC): A survey of self-help support groups in health care. Health Expectations 18(4), 562-577.

Hui D., Bruera E. (2016). Integrating palliative care into the trajectory of cancer care. Nature Reviews Clinical Oncology 13(3), 159-171.

Javier A.D., Figueroa R., Siew E.D., et al. (2017). Reliaiblty and utility of the surprise question in CKD stages 4 to 5. American Journal of Kidney Disease 70(1), 93-101. 
Maxwell A., Ozmen M., Iezzi A., et al. (2016). Deriving population norms for the AQoL-6D and AQoL-8D multi-attribute utility instruments from web-based data. Quality of Life Research 25(12), 3209-3219.

Morton R.L., Webster A.C., McGeechan K., et al. (2016). Conservative management and end of life care in an Australian cohort with ESRD. Clinical Journal of the American Society of Nephrology, 11(12), 2195-2203.

Murtagh F.E., Addington-Hall J.M., Edmonds P.M., et al. (2007). Symptoms in advanced renal disease: A cross-sectional survey of symptom prevalence in stage 5 chronic kidney disease managed without dialysis. Journal of Palliative Medicine 10(6), 126676.

Murtagh F.E.M., Burns A., Moranne O., et al. (2016). Supportive care: Comprehensive conservative care in end-stage kidney disease. Clinical Journal of the American Society of Nephrology 11, 1909-1914.

Ngu K., Reid D., Tobin A. (2017). Trends and outcomes of chronic kidney disease in intensive care: A 5-year study. Internal Medicine Journal 47(1), 62-67.

Richardson J., Peacock S., Hawthorne G., et al. (2012). Construction of the descriptive system for the assessment of quality of life AQoL-6D utility instrument. Health and Quality of Life Outcomes 10(38), 1-9.

Rosenwax L., Spilsbury K., McNamara BA., et al. (2016). A retrospective population based cohort study of access to specialist palliative care in the last year of life: Who is still missing out a decade on?" BMC Palliative Care 15(1), 46.

Schwartz C.E. \& Sprangers M.A. (1999). Methodological approaches for assessing response shift in longitudinal health-related qualty-of-life research. Social Sciences and Medicine 48, 1531-1548.

Singer, A. E., Goebel J. R., Kim Y. S., et al. (2016). "Populations and Interventions for Palliative and End-of-Life Care: A Systematic Review." Journal of Palliative Medicine 9(9), 1-14.

Solano JP., Gomes B. \& Higginson IJ. (2006). A comparison of symptom prevalence in far advanced cancer, AIDS, heart disease, chronic obstructive pulmonary disease and renal disease. Jounral of Pain and Symptom Management 31(1), 58-69.

Tan T., Brennan F., Brown MA. (2017). Impact of dialysis on symptom burden and functional state in the elderly. Renal Society of Australasia Journal 13(1), 22-30.

Webster AC., Nagler EV., Morton RL., et al. (2017). Chronic Kidney Disease. The Lancet 389(10075), 1238-1252.

World Health Organisation. (2016). Cancer: WHO Definition of Palliative Care. Accessed September 2, 2016. http://www.who.int/cancer/palliative/definition/en/. 
World Health Organisation Executive Board. (2013). Strengthening of Palliative Care as a Component of Integrated Treatment Throughout the Life Course. Accessed February 13, 2014. http://apps.who.int/gb/ebwha/pdf files/EB134/B134_28-en.pdf.

Zeng L., Bedard G., Cella D., et al. (2013). Preliminary results of the generation of a shortened quality of life assessment for patients with advanced cancer: The FACITPal-14. Journal of Palliative Medicine 16(5), 509-515. 